\title{
Modified Variational Mode Decomposition for Power Line Interference Removal in ECG Signals
}

\author{
Neethu Mohan*, Sachin Kumar S*, Prabaharan Poornachandran**, K.P Soman* \\ * Centre for Excellence in Computational Engineering and Networking, Amrita Vishwa Vidyapeetham, India \\ ** Amrita Center for Cybersecurity Systems and Networks, Amrita Vishwa Vidyapeetham, India
}

\begin{tabular}{l} 
Article Info \\
\hline Article history: \\
Received Jul 14, 2015 \\
Revised Nov 11, 2015 \\
Accepted Nov 30, 2015 \\
\hline Keyword: \\
ECG signals \\
Mode fixing \\
Modified variational mode \\
decomposition \\
Power line interference removal
\end{tabular}

\begin{abstract}
Power line interferences (PLI) occurring at 50/60 $\mathrm{Hz}$ can corrupt the biomedical recordings like ECG signals and which leads to an improper diagnosis of disease conditions. Proper interference cancellation techniques are therefore required for the removal of these power line disturbances from biomedical recordings. The non-linear time varying characteristics of biomedical signals make the interference removal a difficult task without compromising the actual signal characteristics. In this paper, a modified variational mode decomposition based approach is proposed for PLI removal from the ECG signals. In this approach, the central frequency of an intrinsic mode function is fixed corresponding to the normalized power line disturbance frequency. The experimental results show that the PLI interference is exactly captured both in magnitude and phase and are removed. The proposed approach is experimented with ECG signal records from MIT-BIH Arrhythmia database and compared with traditional notch filtering.
\end{abstract}

Copyright (C) 2016 Institute of Advanced Engineering and Science. All rights reserved.

\section{Corresponding Author:}

Neethu Mohan,

Centre for Excellence in Computational Engineering and Networking,

Amrita Vishwa Vidyapeetham,

Coimbatore, India-64112

Email: neethumohan.ndkm@gmail.com

\section{INTRODUCTION}

Biomedical recordings are usually corrupted with power line disturbances and which results an erroneous data analysis. The main causes of power line disturbances in biomedical recordings are capacitive and magnetic coupling to building power lines and to non power line noise sources, nearby electrical appliances and mains wiring [1], [2]. The power line interference contains the fundamental frequency component at 50/60 Hz along with higher order harmonics. The removal of these interferences from the biomedical recordings is a complicated task since the behaviour of these disturbances is non stationary in nature. The PLI cancellation is important for proper interpretation of neural signals.

Several approaches have been proposed for the removal of power line interferences in biomedical recordings. The classical approach for removing power line disturbances is using a notch filter [3], [4]. But this filtering approach is not efficient due to the non stationary nature of the interferences and also due to frequency variations in the signal. Another common technique for interference cancellation is based on spectrum estimation but for real time data analysis this method is found inadequate [5]. Later several adaptive interference cancellation approaches have been proposed in various articles. In [6], proposes an algorithm based on Adaptive Notch Filter (ANF) approach for fundamental frequency estimation. Later the harmonics are estimated using discrete-time oscillators and then the amplitude and phase are measured using a simple recursive least square (RLS) algorithm. A discrete-time linear Kalman Notch filter based approach is used for PLI removal in [7]. Since the filter design is linear, this approach does not require any information 
about the phase and amplitude of the interferences. Lattice based second order infinite impulse response (IIR) notch filter is proposed in [8] for power line noise removal. Maniruzzaman et al, designed an adaptive filter based on least-mean-square concept for the removal of PLI from ECG recordings [9]. An Alpha-Beta filter based approach is used in [10]. An adaptive interference canceller with a second order PLL is proposed in [11]. The PLL can help to handle with the frequency deviations in the interference. This system is insensitive to baseline fluctuations and large amplitude variations. Weiner-Hopf equation can be used for finding the initial condition of the filter in [12] and based on that an adaptive system is designed for interference removal. Various signal processing algorithms are also employed for ECG noise removal [13-20]. In [13], Mateo et al, utilized the adaptability of Artificial Neural Network (ANN) algorithm to the time varying, non linear features of ECG signals for interference removal. A sliding DFT based phase locking scheme is proposed in [14]. A least mean square based adaptive interference canceller is designed by replacing the squared-error at each sample by mean-square-error of an error vector in the LMS algorithm [15]. It is a modified version of the existing adaptive canceller included with error estimation in the neighbouring samples. A State Space Recursive Least Square (SSRLS) technique is employed for PLI removal in [16], [17]. The main advantage of this method is it does not require a separate reference power line for tracking the PLI. An FFT based algorithm for finding the central frequency of power line is used in [18]. Then subtracting the noise estimated from the corrupted signal for interference cancellation. Empirical mode decomposition (EMD) combined with filter approach is used for PLI removal in [19]. EMD is a data- driven adaptive signal decomposition algorithm and is used for capturing of power line noise in one of the IMF.
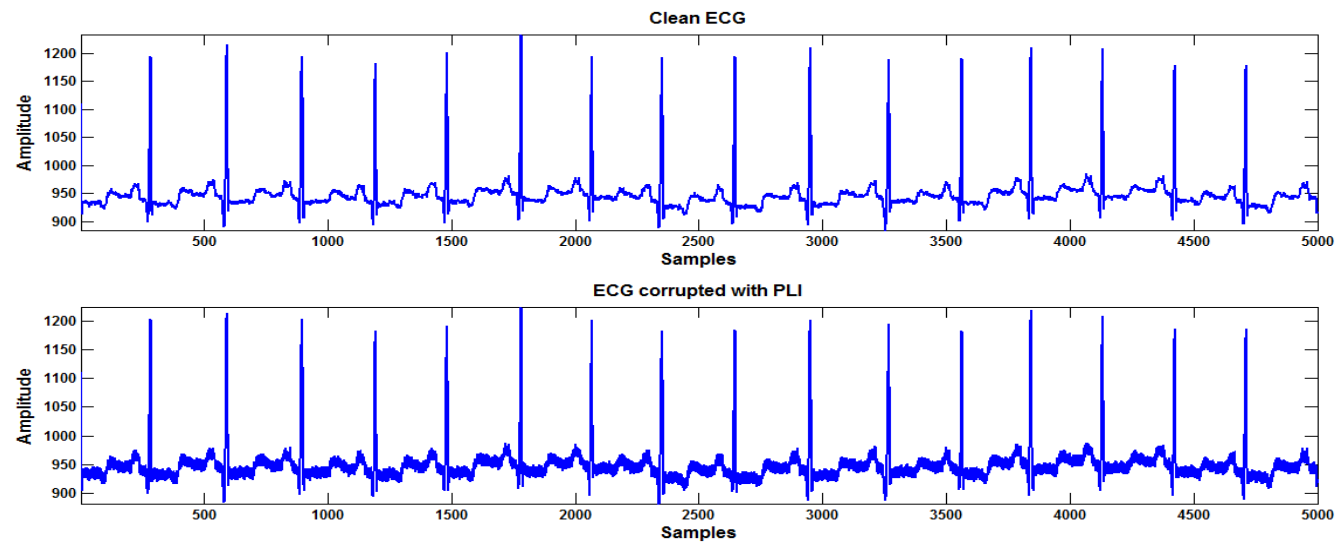

Figure 1. Clean ECG signal and ECG corrupted with PLI

In this paper, modified variational mode decomposition (VMD), based approach for interference removal is discussed with experimental evaluation on noisy ECG data. Variational mode decomposition uses the concept of calculus of variation with Alternating Direction Method of Multipliers (ADMM) for determining the various modes present in the signal. The remaining section of the paper is organized as follows - section 2 describes the variational mode decomposition followed by the proposed approach for PLI removal. Section 3 describes the performance evaluation of the proposed approach on ECG signals and comparison with conventional notch filtering. Section 4 concludes the paper.

\section{PROPOSED APPROACH}

The proposed approach uses modified variational mode decomposition for power line disturbance cancellation. The concept of VMD and how it is utilized for efficient power line noise cancellation is discussed in this section.

\subsection{Variational Mode Decomposition}

The concept of variational mode decomposition is proposed in [20]. VMD, decomposes the signal into various modes or intrinsic mode functions (IMF's) using calculus of variation. Each mode of the signal is assumed to have compact frequency support around a central frequency. VMD tries to find out these central frequencies and IMF's centered on those frequencies concurrently using an optimization methodology called Alternating Direction Method of Multipliers (ADMM). In VMD, a function that can measure the 
bandwidth of IMF, $u_{k}(t)$ is calculated. For that, first compute Hilbert transform of IMF, $u_{k}^{H}(t)$ and formulate an analytic function $\left(u_{k}(t)+j u_{k}^{H}(t)\right)$. The frequency spectrum of this function is one sided and assumed to be centered on $\omega_{k}$. By multiplying this analytical signal with $e^{-j \omega_{k} t}$, the signal is frequency translated to be centered at origin. The integral of the square of the time derivative of this frequency translated signal is a measure of bandwidth of the IMF, $u_{k}(t)$. Now the problem can be formulated as an optimization problem as follows,

$$
\begin{aligned}
& \min _{u_{k}, \omega_{k}} \sum_{k}\left\|\partial_{t}\left[\left(\left(\delta(t)+\frac{j}{\pi t}\right) * u_{k}(t)\right) e^{-j \omega_{k} t}\right]\right\|_{2}^{2} \\
& \text { s.t } \quad \sum_{k} u_{k}=f
\end{aligned}
$$

Where $f$ is the original signal. That is the sum of the bandwidths of $k$ modes is minimized subject to the condition that sum of the $k$ modes is equal to the original signal. So the algorithm tries to find out $k$ unknown central frequencies and $k$ functions centered at those frequencies. Now this constrained optimization problem is converted into an unconstrained problem using the augmented Lagrangian multiplier method. The augmented Lagrangian multiplier corresponds to the above optimization is as follows;

$$
L\left(u_{k}, \omega_{k}, \lambda\right)=\alpha \sum_{k}\left\|\partial_{t}\left[\left(\left(\delta(t)+\frac{j}{\pi t}\right) * u_{k}(t)\right) e^{-j \omega_{k} t}\right]\right\|_{2}^{2}+\left\|f-\sum_{k} u_{k}\right\|_{2}^{2}+\left\langle\lambda, f-\sum_{k} u_{k}\right\rangle
$$

Now this can be solved via the ADMM framework and the corresponding update equations are obtained. In ADMM, solve for one variable at a time assuming that all the other variables are known. The update for IMF, $u_{k}(t)$ is,

$$
\hat{u}_{k}^{n+1}=\left(\hat{f}-\sum_{i \neq k} \hat{u}_{i}\right) \frac{1}{\left(1+2\left(\omega-\omega_{k}\right)^{2}\right)} \quad, \omega \geq 0
$$

The modes are updated in the frequency domain. The update equation for central frequency $\omega_{k}$ is,

$$
\omega_{k}^{n+1}=\frac{\int_{0}^{\infty} \omega\left|\hat{u}_{k}(\omega)\right|^{2} d \omega}{\int_{0}^{\infty}\left|\hat{u}_{k}(\omega)\right|^{2} d \omega}
$$

And update for $\lambda$ is,

$$
\lambda^{n+1} \leftarrow \lambda^{n}+\tau\left(f-\hat{u}_{k}^{n+1}(t)\right)
$$

The Lagrangian multiplier $\lambda$ is for exact reconstruction and is updated as dual ascent [20].

\subsection{Modified VMD for PLI Removal}

In this approach, a modified VMD algorithm is proposed for removing the $50 / 60 \mathrm{~Hz}$ power line interferences in ECG records. $\omega$ represents the central frequency corresponding to the IMF's. In the modified algorithm, the $\omega$ is fixed to the normalized frequency corresponding to $50 / 60 \mathrm{~Hz}$. The $\omega$ varies in the range from 0 to $\pi$. In the updating procedure, the $\omega$ that we have fixed remains the same and the remaining $\omega$ will be updated each time. The modes corresponding to each $\omega$ will get updated until the algorithm converges. As the frequency of the power line interference is $50 / 60 \mathrm{~Hz}$, the mode fixed with the corresponding normalized frequency will capture this power line disturbance. All other frequency components presents in the signal will be captured by other IMF's. Similar to the original theory [20], combining all the IMF's gives original signal. The error between the original signal and the sum of all modes is negligible. Now, by removing the mode with PLI disturbance, the reconstructed signal results in a power line interference free signal. One of the main issues with the original VMD algorithm is that, if the component of interest is of less

Modified Variational Mode Decomposition for Power Line Interference Removal in ... (Neethu Mohan) 
power compared to other components present in the signal, VMD could not remove it. This problem is avoided in the modified VMD algorithm. In this algorithm, by fixing the central frequency, we are able to capture the low power components also. By this same way, we can extract higher order power harmonics present in the signal. During this operation, it must be observed that the nearby frequencies are not all affected. So the proposed methodology, acts as a very sharp notch filter to remove the specified frequency.

\section{Modified VMD algorithm}

1. Initialize $\hat{u}_{k}^{1}, \hat{\omega}_{k}^{1}, \hat{\lambda}_{k}^{1}, n \leftarrow 0$

2. Initialize $\hat{\omega}_{2}$ as the normalized frequency corresponding to $50 / 60 \mathrm{~Hz}$

Repeat

$n \leftarrow n+1$

3. for $k=1: K$ do

4. update $\hat{u}_{k}$ for all $\omega \geq 0$

$$
\hat{u}_{k}^{n+1} \leftarrow \frac{\hat{f}-\sum_{i<k} \hat{u}_{i}^{n+1}-\sum_{i>k} \hat{u}_{i}^{n}+\frac{\hat{\lambda}^{n}}{2}}{1+2 \alpha\left(\omega-\omega_{k}^{n}\right)^{2}}
$$

5. update $\omega_{k}$ for all $\omega \geq 0$

6. if $k=2$, then no update of $\omega$

else

$$
\omega_{k}^{n+1} \leftarrow \frac{\int_{0}^{\omega} \omega\left|\hat{u}_{k}^{n+1}(\omega)\right|^{2} d \omega}{\int_{0}^{\omega}\left|\hat{u}_{k}^{n+1}(\omega)\right|^{2} d \omega}
$$

7. end

8. Dual ascent for all $\omega \geq 0$

$$
\hat{\lambda}^{n+1} \leftarrow \hat{\lambda}^{n}+\tau\left(\hat{f}-\sum_{k} \hat{u}_{k}^{n+1}\right)
$$

9. until convergence: $\sum_{k}\left\|\hat{u}_{k}^{n+1}-\hat{u}_{k}^{n}\right\|_{2}^{2} /\left\|\hat{u}_{k}^{n}\right\|_{2}^{2}<\varepsilon$

\section{RESULTS AND DISCUSSION}

The performance of the proposed approach is evaluated with MIT-BIH Arrhythmia database [21]. The ECG signal records containing power line disturbances at $60 \mathrm{~Hz}$ is considered for the experimenting purpose. It does not contain any harmonics. In figure 2, it shows a frame of ECG record 228 with $60 \mathrm{~Hz}$ power disturbance along with its power spectral density (PSD) plot. From the PSD plot, the presence of 60 $\mathrm{Hz}$ power disturbances can be seen as a small lobe at $60 \mathrm{~Hz}$.

This ECG signal is then given to modified VMD algorithm, where the second mode is fixed with normalized frequency of $60 \mathrm{~Hz}$. VMD creates IMF's based on the parameters such as total number of modes, bandwidth constraint, time step of the dual ascent etc. The modes thus obtained are shown in figure 3 . The second mode is made to operate for $60 \mathrm{~Hz}$. The power disturbances at $60 \mathrm{~Hz}$ will get captured in the same mode. This can be clearly visible from figure 3. It is observed that the reconstructed signal has some loss of height (or magnitude) at the location where there are R-peaks. The heights of R-peaks are really crucial for analysis purpose. The lost magnitude of the peak can be seen in the mode corresponding to interference. So a thresholding step is introduced in the proposed methodology to avoid the loss of information. The importance of thresholding can be understood by observing the small peak like signal samples in the second mode at several locations. These locations are marked with red rings in figure 3 . Hence, by finding a threshold as an average on energy, those small peaks can be extracted, and is added back to the reconstructed signal. Now the reconstructed signal will be the clear ECG signal without PLI. Figure 4 shows PSD of PLI removed reconstructed ECG signal. From the PSD it can be observed that the lobe at $60 \mathrm{~Hz}$ is not present. This exhibits the power of modified VMD algorithm to exactly take out the specified frequency and the nearby frequencies are not all affected. 
Consider another scenario, where the ECG signal contains $50 \mathrm{~Hz}$ power line noises along with harmonics in it. In this case, the second mode of VMD algorithm is fixed for capturing frequencies of $50 \mathrm{~Hz}$ and the third mode is fixed for capturing the odd harmonics of $150 \mathrm{~Hz}$ present in the signal. The signal and its PSD plot are given in figure 5. The corresponding modes obtained are given in figure 6. From the modes obtained, it is observed that the second and third modes captured the power line fundamental frequency and the odd harmonics present in the noisy ECG signal exactly. Hence, for reconstruction, removing those modes will result in a PLI free signal. Figure 7 shows the noisy and noise removed ECG signals along with the PSD plot.
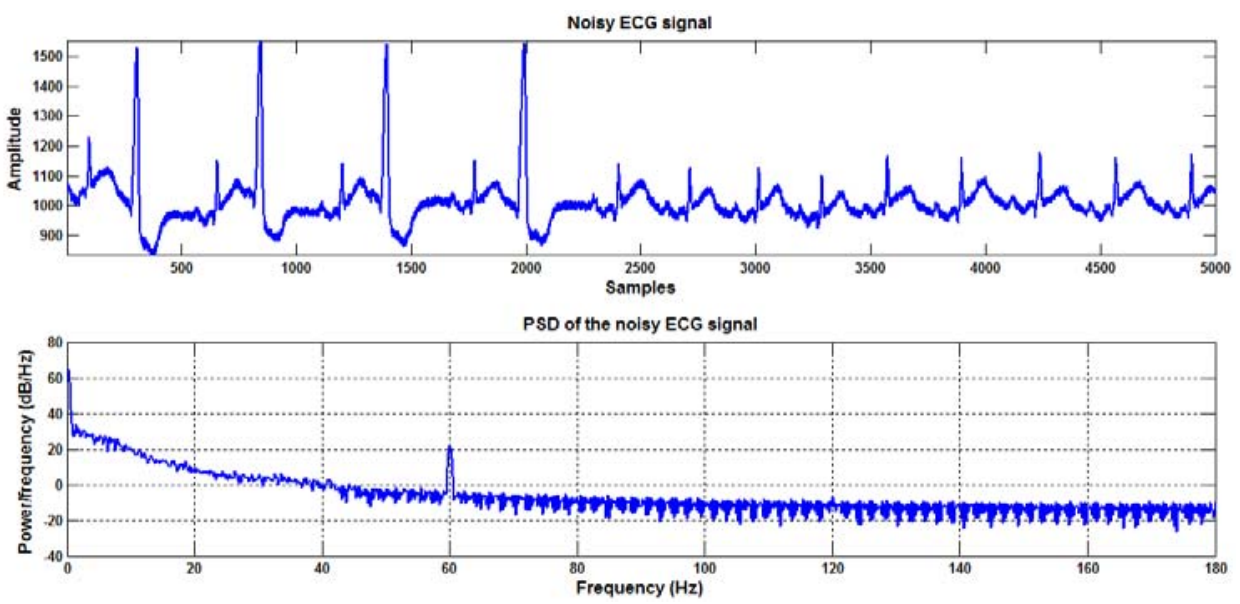

Figure 2. ECG signal with PLI at $60 \mathrm{~Hz}$ and corresponding PSD
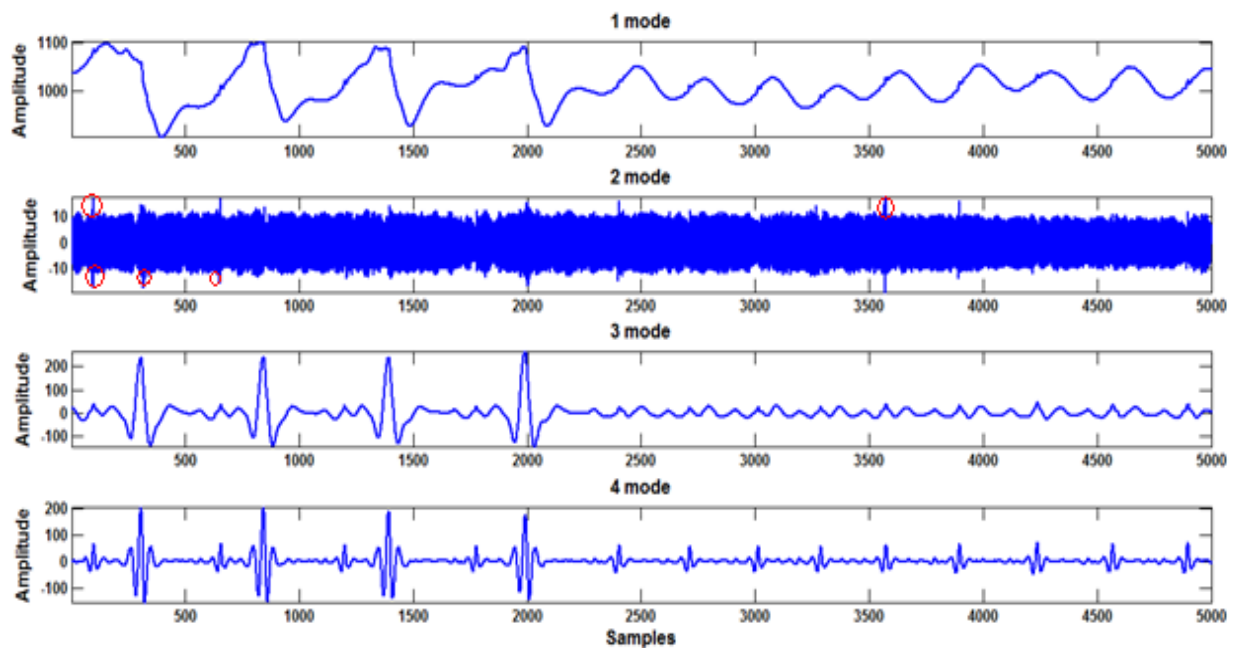

Figure 3. IMF’s obtained through modified VMD of record 228

The proposed modified VMD algorithm is compared with the conventional notch filtering approach. In notch filtering, when the interference fundamental frequency is slightly deviated from the $50 / 60 \mathrm{~Hz}$, the notch filters fail to remove the interference present in the signal. From the experiments, it can also observed that the notch filters completely fails to pick up the deviations occuring in higher order harmonics of the interference. However the proposed algorithm successfully removes the interferences under both situations. Figure 8.a and 8.b represents the PSD plot of the proposed modified VMD algorithm and figure 8.c and 8.d represents the traditional notch filtering PSD plot. From the plots it can be seen that the notch filtering completely fails to pick up the interference when the fundamental frequency is slightly deviated from $60 \mathrm{~Hz}$. Where as the proposed approach successfully removes the interference present in the signal. 

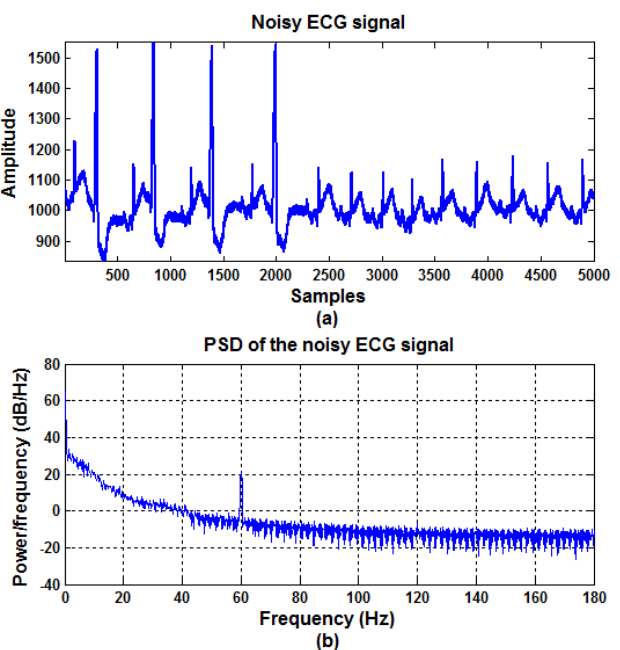

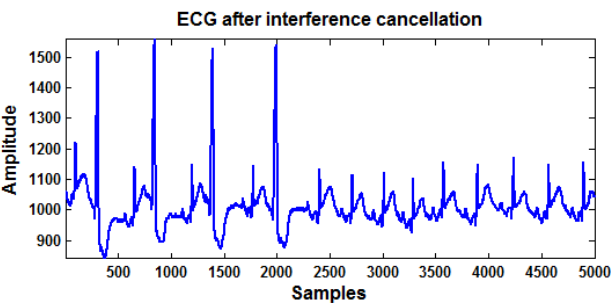

$$
\begin{aligned}
& \text { Sample } \\
& \text { (c) }
\end{aligned}
$$

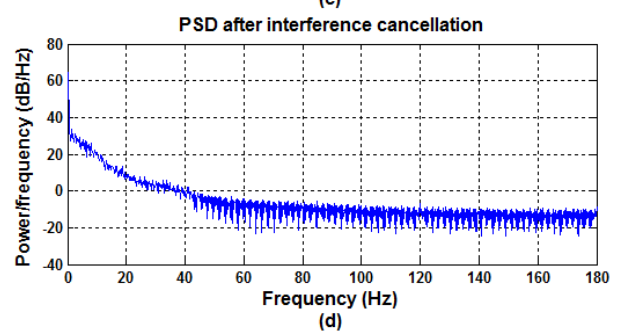

Figure 4. (a), (b) Noisy record 228 and its PSD; (c), (d) denoised record 228 and PSD of denoised portion
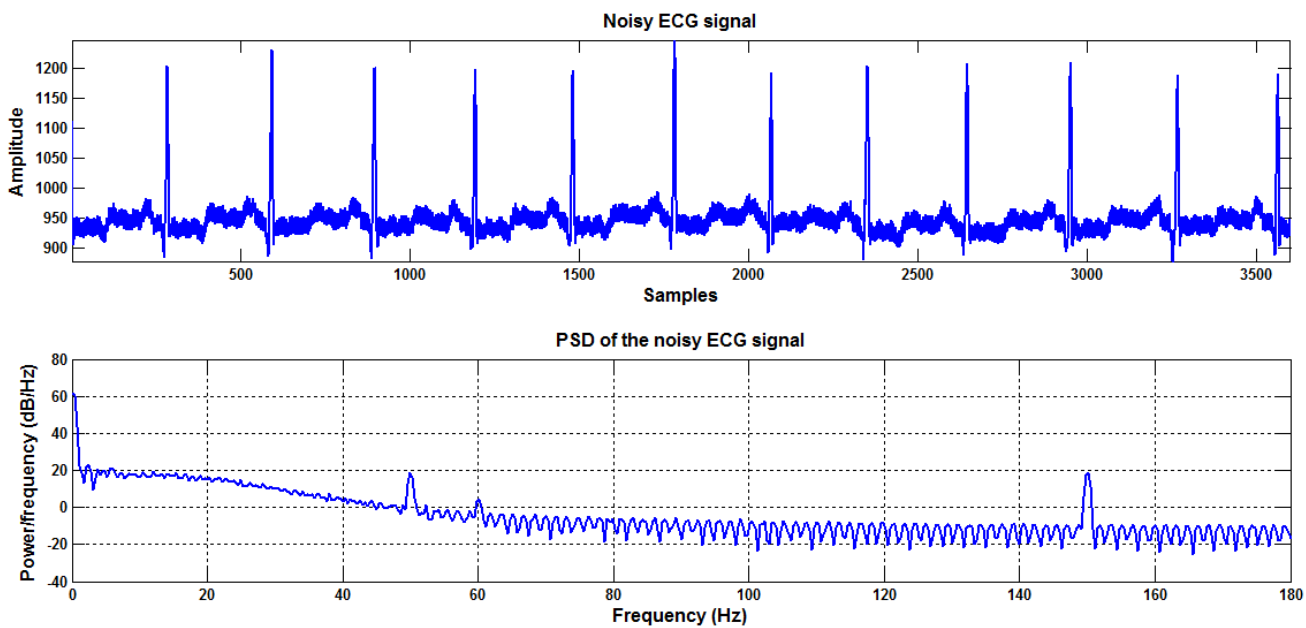

Figure 5. ECG signal with PLI at $50 \mathrm{~Hz}$ and its harmonics and corresponding PSD of the signal
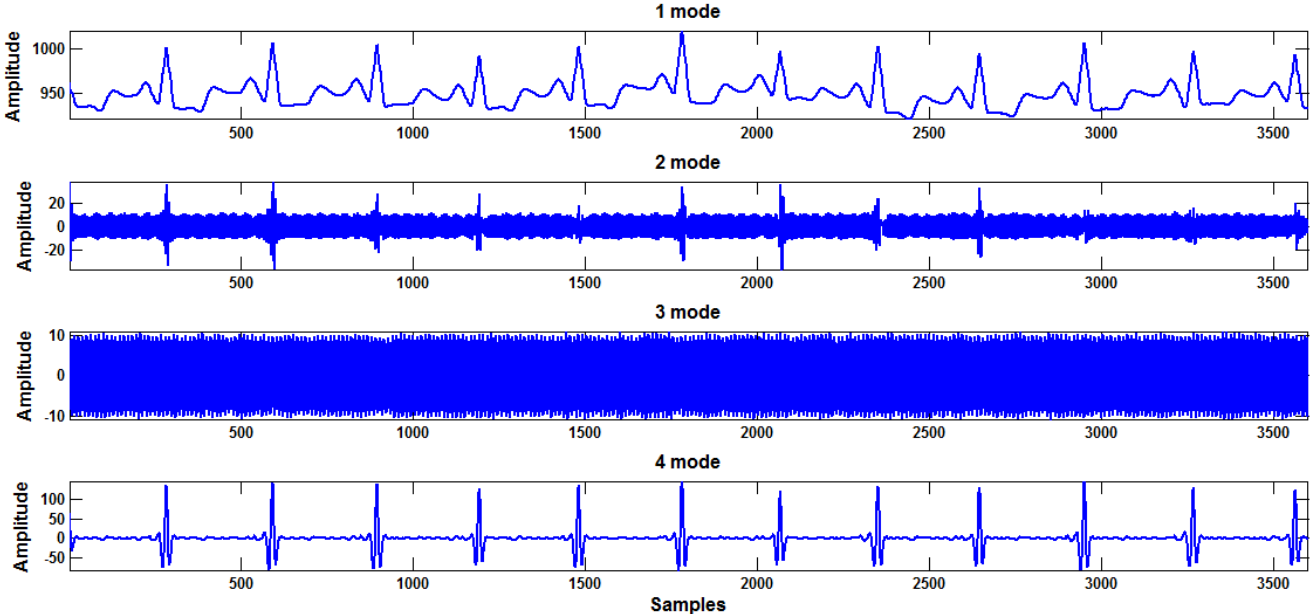

Figure 6. Modes obtained through modified VMD 


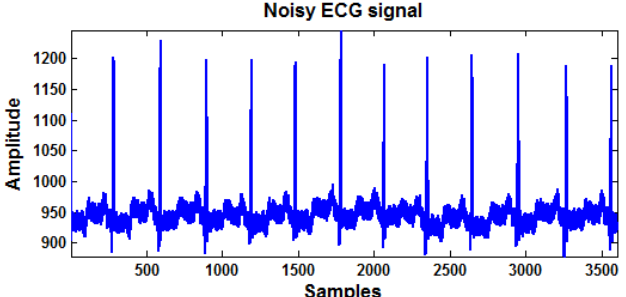

(a)

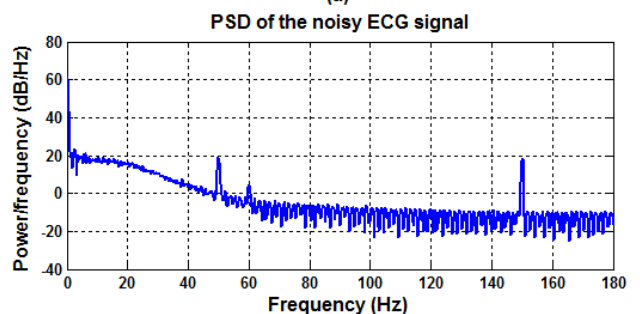

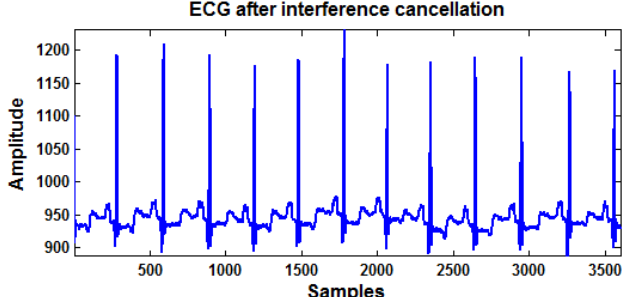

(c)

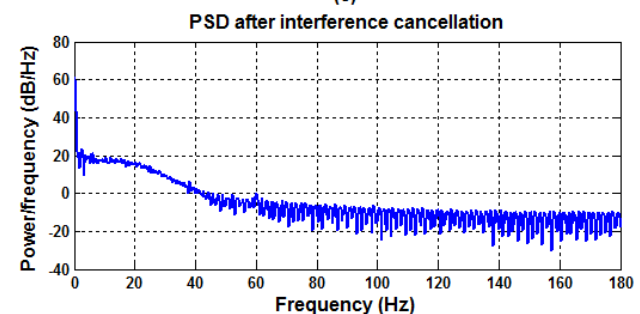

Figure 7. (a), (b) Noisy ECG signal and its PSD; (c), (d) ECG signal after noise cancellation and its PSD

\subsection{Signal to Noise Ratio}

The performance of the proposed approach is also evaluated in terms of input and output signal to noise ratio (SNR). The input SNR ( $\mathrm{SNR}_{\text {in }}$ ) is calculated by finding the ratio of the power of the clean ECG signal to the power of the interference signal. When the power line disturbance increases, the value of input SNR will be low. The output SNR $\left(\mathrm{SNR}_{\text {out }}\right)$ is calculated by finding the ratio of the power of the estimated signal to the power of the error in the estimation. The correctness of the proposed approach is checked by varying the $\mathrm{SNR}_{\text {in }}$ from $-10 \mathrm{~dB}$ to $30 \mathrm{~dB}$ and the corresponding $\mathrm{SNR}_{\text {out }}$ values are tabulated in Table 1 . To do the experiments, ECG signal record 101 of MIT-BIH Arrhythmia database is chosen. While it was observed that, for low $\mathrm{SNR}_{\text {in }}$ signal, the last mode of the VMD doesn't contain any signal information. Hence, during reconstruction, avoiding this mode gives a signal with improved $\mathrm{SNR}_{\text {out }}$.

Table 1. Results of evaluation on ECG record 101

\begin{tabular}{ccc}
\hline Signal Record & SNR $_{\text {in }}$ & SNR $_{\text {out }}$ \\
\hline 101 & 28.6982 & 49.8585 \\
101 & 22.6776 & 47.6108 \\
101 & 16.6570 & 44.0847 \\
101 & 11.7963 & 30.5947 \\
101 & 2.6776 & 22.6983 \\
101 & -0.8442 & 20.5207 \\
101 & -6.8648 & 14.6240 \\
101 & -9.3636 & 12.1169 \\
\hline
\end{tabular}



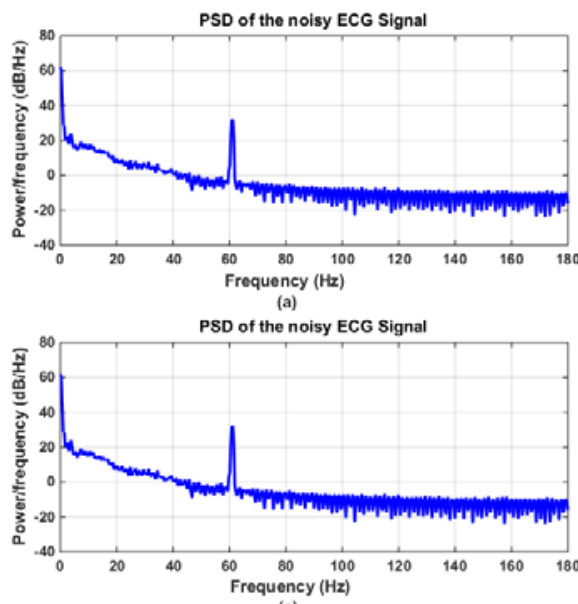
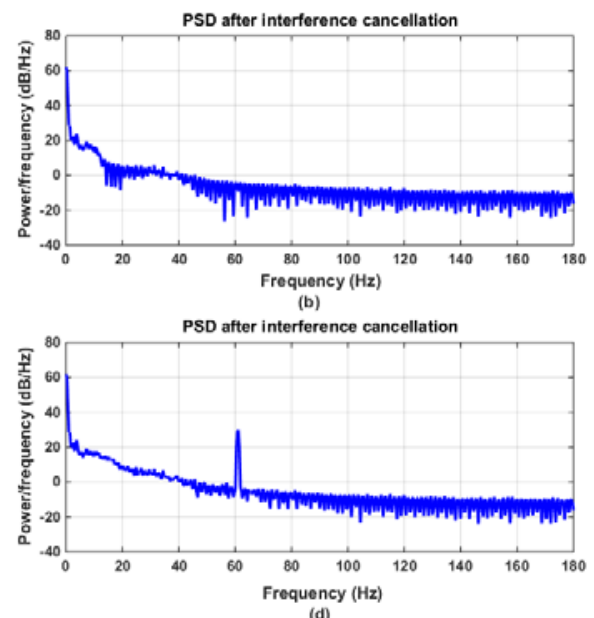

(d)

Figure 8. (a), (b) Performance of modified VMD algorithm; (c), (d) Performance of traditional notch filter

\section{CONCLUSION}

The proposed approach using modified variational mode decomposition clearly removes the 50/60 $\mathrm{Hz}$ interference and its harmonics from the ECG recordings. By correctly fixing the boundaries for the signal in VMD mode calculation, it accurately extracts the specified frequency, without altering the nearby frequencies. During the reconstruction of signal by excluding the mode that captured the noise and harmonics, it is shown that the reconstructed signal have a high $\mathrm{SNR}_{\text {out }}$ compared to $\mathrm{SNR}_{\mathrm{in}}$. From the observations made it can be concluded that, the proposed modified VMD based approach is appropriate for power line interference cancellation from ECG signals. It can also conclude that the proposed appraoch acts as a very sharp notch filter by removing the specified frequency.

\section{REFERENCES}

[1] C.K. Thorp, et al, "Interference and noise in human intracranial microwire recordings", IEEE Transactions on Biomedical Engineering, vol. 56, pp. 30-36, 2009.

[2] M. F. Chimene, et al, "Comprehensive model for power line interference in biopotential measurements", IEEE Transactions on Instrumentation and Measurement. vol. 49, pp. 535-540, 2000.

[3] M. Ferdjallah, et al, "Adaptive digital notch filter design on the unit circle for the removal of powerline noise from biomedical signals", IEEE Transactions on Biomedical Engineering. vol. 41, pp. 529-536, 1994.

[4] A. Nehorai, et al, "A minimal parameter adaptive notch filter with constrained poles and zeros", IEEE Transactions on Acoustics, Speech and Signal Processing. vol. 33, pp. 983-996, 1985.

[5] Mitra, P., Bokil, H. et al, "Observed brain dynamics", Oxford University Press. 2007.

[6] M.R. Keshtkaran, et al, "A fast, robust algorithm for power line interference cancellation in neural recording", Journal of neural engineering. vol. 11, pp. 026017, 2014.

[7] R. Sameni, et al, "A linear kalman notch filter for power-line interference cancellation", 16th CSI International Symposium on Artificial Intelligence and Signal Processing (AISP). pp. 604-610, 2012.

[8] S.S. Dhillon, et al, "Power line interference removal from electrocardiogram using a simplified lattice based adaptive IIR notch filter", 23rd Annual International Conference of the Engineering in Medicine and Biology Society. vol. 4, pp. 3407-3412, 2001.

[9] M. Maniruzzaman, et al, "Least-Mean-Square algorithm based adaptive filters for removing power line interference from ECG signal", IEEE International Conference on Informatics, Electronics \& Vision (ICIEV). pp. 737-740, 2012.

[10] Jamwal, S. et al, "Power line interference cancellation in ECG signals using Alpha-Beta filter", IEEE International Conference on Signal Processing, Computing and Control (ISPCC). pp. 1-6, 2012.

[11] S.M. Martens, et al, "An improved adaptive power line interference canceller for electrocardiography", IEEE Transactions on Biomedical Engineering. vol. 53, pp. 2220-2231, 2006.

[12] A. Manosueb, et al, "PLI Cancellation in ECG Signal Based on Adaptive Filter by Using Wiener-Hopf Equation for Providing Initial Condition", Computational and mathematical methods in medicine. 2014.

[13] J. Mateo, et al, "Neural network based canceller for powerline interference in ECG signals", IEEE Computers in Cardiology. pp. 1073-1076, 2008.

[14] S. Mishra, et al, "A Power-Line Interference Canceler Based on Sliding DFT Phase Locking Scheme for ECG Signals", IEEE Transactions on Indumenstration and Measurement, vol. 64, pp. 132-142, 2015. 
[15] H.N. Bharath, et al, "A new LMS based adaptive interference canceller for ECG power line removal", IEEE International Conference on Biomedical Engineering (ICoBE). pp. 68-73, 2012.

[16] M. Butt, et al, "Power Line Interference tracking in ECG signal using State Space RLS", 8th IEEE Conference on Industrial Electronics and Applications (ICIEA), pp. 211-215, 2013.

[17] N. Razzaq, et al, "Self tuned SSRLS filter for online tracking and removal of power Line Interference from Electrocardiogram", IEEE International Conference on Modelling, Identification \& Control (ICMIC). pp. 339-343, 2013.

[18] F. Shirbani, et al, "ECG power line interference removal using combination of FFT and adaptive non-linear noise estimator", 21st Iranian Conference on Electrical Engineering (ICEE). pp. 1-5, 2013.

[19] Z. Zhidong, et al, "A novel cancellation method of powerline interference in ECG signal based on EMD and adaptive filter", 11th IEEE International Conference on Communication Technology (ICCT), pp. 517-520, 2008.

[20] K. Dragomiretskiy, et al, "Variational mode decomposition", IEEE Transactions on Signal Processing. vol. 62, pp. 531-544, 2014.

[21] http://www.physionet.org/physiobank/database/mitdb/ visited on 10/06/2015. 